\title{
Correspondence
}

\section{Variation of peak expiratory flow rate}

Sir,-In their article, Hetzel and Clark ${ }^{1}$ suggest an amplitude of $20 \%$ in a 24 -hour variation of peak expiratory flow rate should be regarded as a useful screening test for asthma. While I entirely sympathise with the view that the measurement of amplitude variation is one of the best ways of demonstrating variability of airway obstruction, this definition certainly includes many patients who would be regarded as bronchitics without any evidence of asthma. In my paper, ${ }^{2}$ it was demonstrated that the mean amplitude of variation of bronchitis, measured in a different way, was well over $20 \%$ before and over $20 \%$ after bronchodilator.

As it could be argued that variation of peak flow rate, at any rate in quite normal subjects, is principally a deviation below a norm, rather than a deviation about a mean, it seems logical to measure amplitude against the norm, rather than the mean. I appreciate that this does not fit the mathematical model of Clark and Hetzel as well, but it could be argued that it does reflect the pathological process more accurately. Calculation of the amplitude against the norm, that is the maximum recorded peak flow rate, results in a lower amplitude than calculation against the mean, as in my papers the calculations of amplitude were made against the minimum achieved.

I have therefore made an approximate recalculation of the data on 508 recordings on approximately 400 patients in the study referred to above and another to be published. ${ }^{3}$ The patients are divided into asthmatics, bronchitics, and patients with features of both conditions. It will be seen (table) that even in pure bronchitics the mean amplitude is above $30 \%$ before bronchodilator and remains at approximately $25 \%$ when measured after bronchodilator.

Table Amplitude of daytime variation of peak expiratory flow rate measured in asthmatics, bronchitics, and in an intermediate group before and after bronchodilators

\begin{tabular}{|c|c|c|c|c|c|c|}
\hline & \multicolumn{2}{|c|}{$\begin{array}{l}\text { Before } \\
\text { bronchodilator }\end{array}$} & \multicolumn{2}{|c|}{$\begin{array}{l}\text { Five minutes } \\
\text { after } \\
\text { bronchodilator }\end{array}$} & \multicolumn{2}{|c|}{$\begin{array}{l}\text { Two hours after } \\
\text { bronchodilator }\end{array}$} \\
\hline & $n$ & mean & $n$ & mean & $n$ & mean \\
\hline Asthma & 82 & 42 & 32 & 26 & 65 & 31 \\
\hline $\begin{array}{l}\text { Intermediate } \\
\text { group }\end{array}$ & 65 & 37 & 28 & 25 & 54 & 30 \\
\hline $\begin{array}{l}\text { Chronic } \\
\text { bronchitics }\end{array}$ & 68 & 31 & 32 & 26 & 82 & 25 \\
\hline
\end{tabular}

These results confirm that there is value in measuring the amplitude of variation of peak flow rate in all forms of airway obstruction, but if $20 \%$ is taken as a screening test for asthma then many patients who are not usually regarded as having asthma will be included within this definition.

CK CONNOLLY

Friarage Hospital

Northallerton

North Yorkshire

\section{References}

1 Hetzel MR, Clark TJH. Comparisons of normal and asthmatic circadian rhythms in peak expiratory flow rate. Thorax 1980; 35:732-8.

2 Connolly CK. Diurnal rhythms in airway obstruction. $\mathrm{Br} J$ Dis Chest 1978; 73:357-66.

3 Connolly CK. The effect of bronchodilation on diurnal rhythms of airway obstruction. $\mathrm{Br} J \mathrm{Dis}$ Chest 1981; in press.

Sir,-We thank Dr Connolly for his interest in our paper. We have used the method of calculation of diurnal variation in peak expiratory flow rate (PEFR) as a percentage of the highest daily reading in some of our previous work. ${ }^{2} 2$ These studies, however, involved asthma patients with very obvious large amplitude circadian rhythms in PEFR where more critical analysis was unnecessary. This is not the case in our latest paper, where we were attempting to identify a low amplitude circadian rhythm in PEFR in normal subjects. This required an unbiased statistical method to avoid misinterpretation of biological noise as a true rhythm.

Cosinor analysis looks for the best fit of a sinusoid with a 24-hour period to the raw data and avoids bias from assumptions about the timing or magnitude of the highest daily reading; which otherwise needs very frequent readings to measure it accurately. We accept that the PEFR rythm is not a perfect sinusoid but this is the best mathematical model which can be submitted to unbiased analysis. Amplitude is best expressed as a percentage of the mean, but is easily converted to a percentage of the highest daily reading by the equation:

$$
d=\frac{200 \times a}{(200+a)}
$$

where $\mathrm{d}=$ amplitude as percent of highest daily reading

$a=$ amplitude (peak to trough) as percent of mean

There is little difference between values for a and $d$ at amplitudes below $30 \% .^{3}$

We have not studied PEFR rhythms in chronic bronchitis, but would not expect patients with chronic obstructive bronchitis without asthma to have larger amplitude PEFR rhythms than normal subjects. 
Clearly our two methods of analysis are not entirely compatible and results of cosinor analysis will give more conservative estimates of amplitude since it takes a balanced view of all the data points whereas calculation from the highest observed daily values is biased towards extremes of variation in the raw data. Moreover, patients in Dr Connolly's study ${ }^{4}$ achieved a lower overall PEFR (mean values $1821 \mathrm{~min}^{-1}$ for males and $1271 \mathrm{~min}^{-1}$ in females). At these lower levels the noise to signal ratio is high and amplitude is liable to overestimation in comparison with our subjects with PEFR in the predicted normal range. Unfortunately, these differences between normal subjects and patients constitute an insuperable obstacle to comparison of their PEFR rhythms. Amplitude measured as percenage of the highest daily reading also relies heavily on the accuracy of these few readings whereas cosinor analysis attaches equal weight to all observations.

We, therefore, maintain that an amplitude of $>20 \%$ of the mean value in the PEFR rhythm is a valid threshold above which the diagnosis of asthma should be considered. As a corollary to this, we have previously found that only some $20 \%$ of asthma patients in hospital had amplitudes of $>25 \%$ measured from raw data as percentages of the highest daily reading. ${ }^{1}$ Thus the threshold for a diagnosis of asthma appears to lie in the range $20-25 \%$. This view is supported by the work of Dawkins and Muers ${ }^{5}$ who have used cosinor analysis to study PEFR rhythms in chronic bronchitis and obtained similar results to our normal data.

TJH CLARK MR HETZEL

Brompton Hospital Fulham Road London

\section{References}

1 Hetzel MR, Clark TJH, Branthwaite MA. Asthma: analysis of sudden deaths and ventilatory arrests in hospital. Br Med J 1977; 1:808-11.

2 Clark TJH, Hetzel MR. Diurnal variation of asthma. BrJ Dis Chest 1977; 71:87-92.

3 Hetzel MR. Observations on 24 hour periodicity in asthma. MD thesis, University of London, 1979.

4 Connolly CK. Diurnal rhythms in airway obstruction. Br J Dis Chest 1979; 73:357-66.

5 Dawkins KD, Muers MF. Diurnal variation in airflow obstruction in chronic bronchitis. Thorax 1981 ; in press.

\section{What is the best treatment for early operable small cell carcinoma of the bronchus?}

Sir,-We wish to take issue with much of the factual data presented by Dr Levison in his editorial ${ }^{1}$ and to suggest that the proposed national study is not practical and will fail to determine the role of surgery in operable small cell carcinoma of the bronchus (SCCB).
SCCB does not behave in a uniform manner; there is a considerable variability of response to chemo- $\stackrel{5}{\rightarrow}$ therapy which does not correlate with any obvious histological subtyping. The statement that $95 \%$ of $\frac{}{0}$ SCCB occurs in men is no longer true; more recent $\frac{\bar{N}}{\sim}$ reports contain ratios of three or four men to one $\vec{\nabla}$ woman and in the last 300 cases treated by our group $40 \%$ were women. The overall incidence is not $36 \%$ o of all lung cancers, but nearer to $20-25 \% .^{2}$

Much of the data presented in the editorial come from studies in patients with "limited" disease but it is $\vec{\omega}$ important to make clear what this term means. It is usually used to describe a category of patients where $\vec{x}$ the disease is confined to one hemithorax and in whom $\dot{\omega}$ there is no detectable metastatic spread. These patients are rarely "operable". In our study 3 sequential staging $\omega$ using CT scanning of thorax and abdomen in 50 con- $\underset{V}{ }$ secutive patients showed that all patients were inoperable, $74 \%$ having $\mathrm{T} 3$ tumours including the three patients who, on chest $\mathrm{x}$-ray, were thought to have T1 $\overrightarrow{-}$ tumours. In our last 315 patients we have seen one operable case. There is now a considerable body of evidence to show that the median survival of even "limited" disease does not exceed nine months when $\vec{\varnothing}$ a local treatment, such as radiotherapy, is used alone. $\stackrel{\infty}{\rightarrow}$ Better results with chemotherapy are achieved in this category of patients, 45 and it is not yet clear if radiotherapy has any part to play in management other than as a palliative procedure. Early results from randomised trials, ${ }^{6}$ including our own, suggest that it does not prolong survival in patients treated adequately with drugs.

After high dose radiation relapse in the chest is common, ${ }^{78}$ indicating that local control is rarely achieved and that the suggested preoperative low dose radiotherapy would not render patients operable. It is not appropriate to draw an analogy between "debulking" surgery in ovarian cancer and small cell car- $\frac{0}{0}$ cinoma. Ovarian cancer often remains localised in the $x$ abdomen and is a slowly growing adenocarcinoma. Small cell carcinoma usually spreads early and grows rapidly. Local surgery is unlikely to influence mortality in this situation.

Cranial irradiation is of no proven benefit for survival in small cell carcinoma and it is not logical to include it in randomised studies until longer survival o is achieved than that currently being obtained.

Dr Levison's proposed study therefore concerns a N tiny minority of patients with small cell carcinoma in N whom the tumour is operable. Adequate investigation $N$ of these patients will probably reveal more extensive $\omega$ disease in many. Furthermore by having four arms to the study, all of which include surgery, it is certain 0 that the investigation will take many years to com- $\frac{\mathscr{D}}{\mathscr{}}$ plete and that the role of surgery will not have been $\stackrel{\odot}{+}$ evaluated. The study design as proposed will assess the $\square$ role of preoperative low dose radiotherapy and brain irradiation. At the present time neither of these aims seems worthwhile.

If a trial of surgery in the rare operable cases is $\stackrel{\square}{\square}$ to be mounted this should include careful preoperative 